\title{
Prediction of unsteady natural convection within a square cavity containing an obstacle at high Rayleigh number value
}

\author{
Basma SOUAYEH ${ }^{1},{ }^{*}$, Nader BEN CHEIKH, Brahim BEN BEYA, Taieb LILI \\ ${ }^{1}$ Laboratory of Mechanic of Fluids, Physics Department, Faculty of Sciences of Tunis, University of \\ Tunis El-Manar, 2092 El-Manar II, Tunis, Tunisia \\ EE-mail address: basmasouayeh@gmail.com
}

Keywords: Natural convection, heating obstacle, Rayleigh number, unsteady state, heat transfer.

ABSTRACT: The present work deals with the prediction of a natural convection flow in a square cavity, partially heated by an obstacle placed at the bottom wall. The two transverse walls and the top wall of the cavity are supposed to be cold, the remaining walls are kept insulated. The main parameter of numerical investigations is the Rayleigh number (engine convection) varying from $10^{3}$ to $10^{5}$. When $\mathrm{Ra}$ is fixed at $10^{7}$, the flow and thermal fields bifurcate and undergoes an unsteady behavior at critical positions.

Flow patterns corresponding to the unsteady state are presented and analyzed in the current study. The simulations were conducted using a numerical approach based on the finite volume method and the projection method, which are implemented in a computer code in order to solve the Navier-Stokes equations.

\section{INTRODUCTION}

Fluid mechanics allows describing a wide variety of natural phenomena $[1,2,3]$. In the current investigation, a numerical study in terms of the problem of natural convection in cavities is carried out. The fluid is considered Newtonian and incompressible, we are mainly interested in two-dimensional cavities incorporating an obstacle on its hot wall. The side walls and the upper wall are maintained at a cold temperature $\mathrm{T}_{\mathrm{c}}$, while the remaining walls are kept adiabatic. In addition, in order to deepen our knowledge about natural convection, a special attention will be considered to investigate the phenomena of the unsteady state $[4,5]$.

\section{DESCRIPTION OF THE MODEL AND NUMERICAL PROCEDURE}

A. Problem formulation

The physical model considered in this work is shown in Figure 1

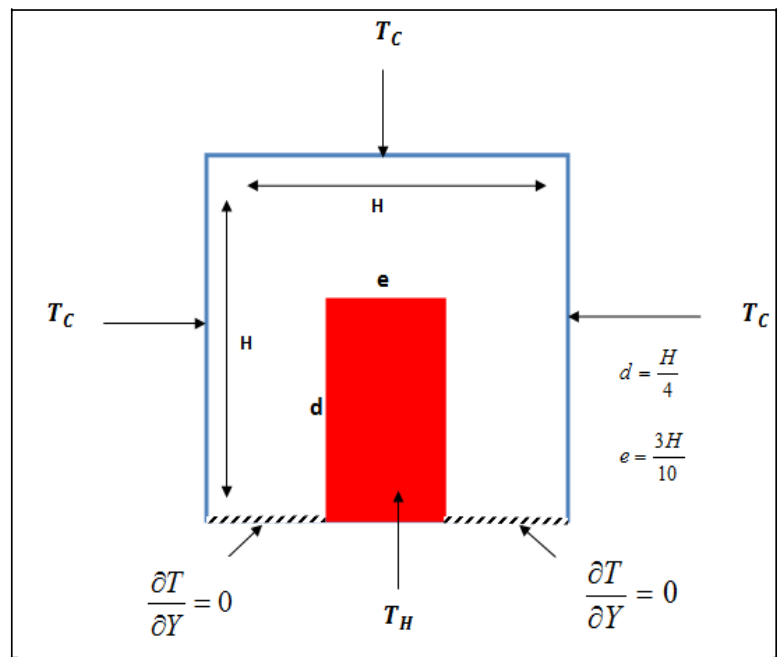

Fig. 1 Physical model and coordinates. 


\section{B. Numerical approach}

The dimensionless governing equations for the present system can be expressed by the following unsteady, two-dimensional equations. In the light of the Boussinesq approximation the dimensionless equation are given by: Continuity:

$$
\frac{\partial u_{i}}{\partial x_{i}}=0 i=1,2
$$

Momentum

$$
\frac{\partial u_{i}}{\partial t}+\frac{\partial\left(u_{i} u_{j}\right)}{\partial x_{j}}=-\frac{\partial p}{\partial x_{i}}+\left(\frac{\operatorname{Pr}}{R a}\right)^{\frac{1}{2}}\left(\frac{\partial^{2} u_{i}}{\partial x_{j} \partial x_{j}}\right)+\theta \delta_{i 2}
$$

Where $\delta_{i 2}$ is the Kronecker symbol and $i=1,2$

Energy:

$$
\frac{\partial \theta}{\partial t}+\frac{\partial\left(u_{i} \theta\right)}{\partial x_{i}}=\left(\frac{1}{R a \operatorname{Pr}}\right)^{\frac{1}{2}} \frac{\partial^{2} \theta}{\partial x_{i} \partial x_{i}}
$$

It is worth noting that equations were solved by an iterative method RBSOR [6] coupled with a multigrid acceleration [7].

\section{RESULTS AND DISCUSSIONS}

\section{A. Effect of Rayleigh number on the unsteady behavior of the flow}

For wide ranges of Rayleigh number $\left(10^{3} \leq \mathrm{Ra} \leq 10^{5}\right)$. It is noted that the global flow pattern is describing a steady state, in contrast the increase of Rayleigh number until $5 \times 10^{5}$ clearly show that the flow become unsteady which is appearing clearly in Figure 2 which represent the evolution of the temperature at the point $(0.1,0.7)$. According to this Rayleigh number value, a slight decrease is observed in the extreme values of the stream function. This decrease is explained by the fact that from $R a=5 \times 10^{5}$, a small areas of recirculation occurs at the horizontal wall of the obstacle. A zoom in this area detect the presence of these two secondary vortices (see Figure 3), which are at the origin of the transition to the unsteady state flow.

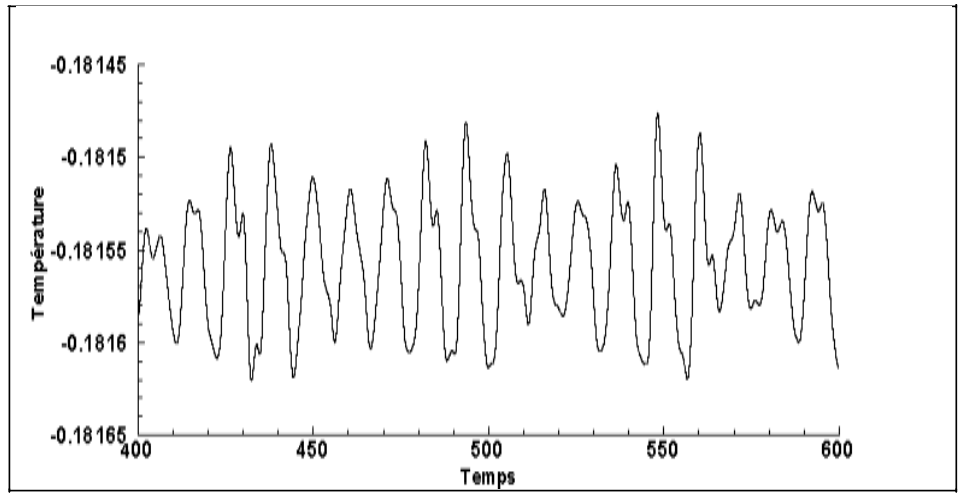

Fig. 2 Evolution of unsteady temperature versus time for $\mathrm{Ra}=5 \times 10^{5}$ and $\mathrm{Pr}=0.71$ 


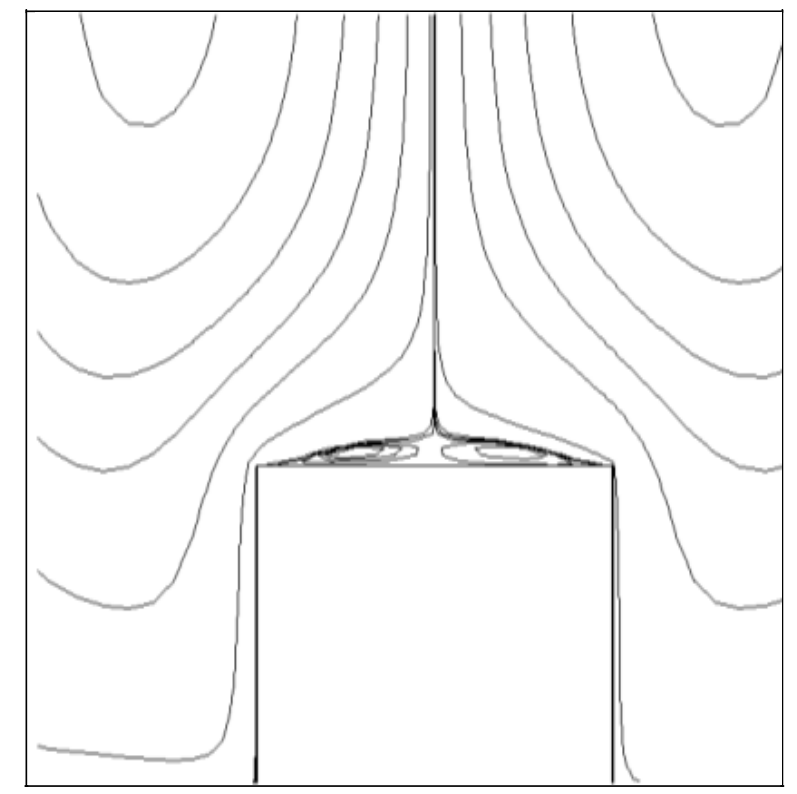

Fig. 3 Zoom of the streamlines at the horizontal wall of the obstacle for $\mathrm{Ra}=5 \times 10^{5}$ and $\operatorname{Pr}=0.71$.

An attention is initially focused on the analysis of unsteady laminar flows. For this purpose, the Rayleigh number is fixed at $10^{7}$ and the results for $R a=5 \times 10^{5}$ were taken as initial solution for the computation of the solution relative to $\mathrm{Ra}=10^{7}$, while the time step used is held fixed at $\mathrm{t}=0.0025$.

Our study is divided into two sections; the first computations are carried out using a dimensionless integration time of 200 , while the second ones correspond to $t$ varying from 200 to 600 .

We now attempt to investigate the effect of Rayleigh number on the flow structure and the heat transfer rate within the cavity for a fixed Prandtl number $\operatorname{Pr}=0.71$ (corresponding to air) and a dimensionless time in the range $(0,200)$. The analysis will be performed by the aid of the practical visualizations of isotherms structures and the velocity component isocontours in order to demonstrate the unsteady character of the flow.

Before you begin to format your paper, first write and save the content as a separate text file. Keep your text and graphic files separate until after the text has been formatted and styled. Do not use hard tabs, and limit use of hard returns to only one return at the end of a paragraph. Do not add any kind of pagination anywhere in the paper. Do not number text heads-the template will do that for you. 
Effect of Rayleigh number (for $0 \leq t \leq 200$ )

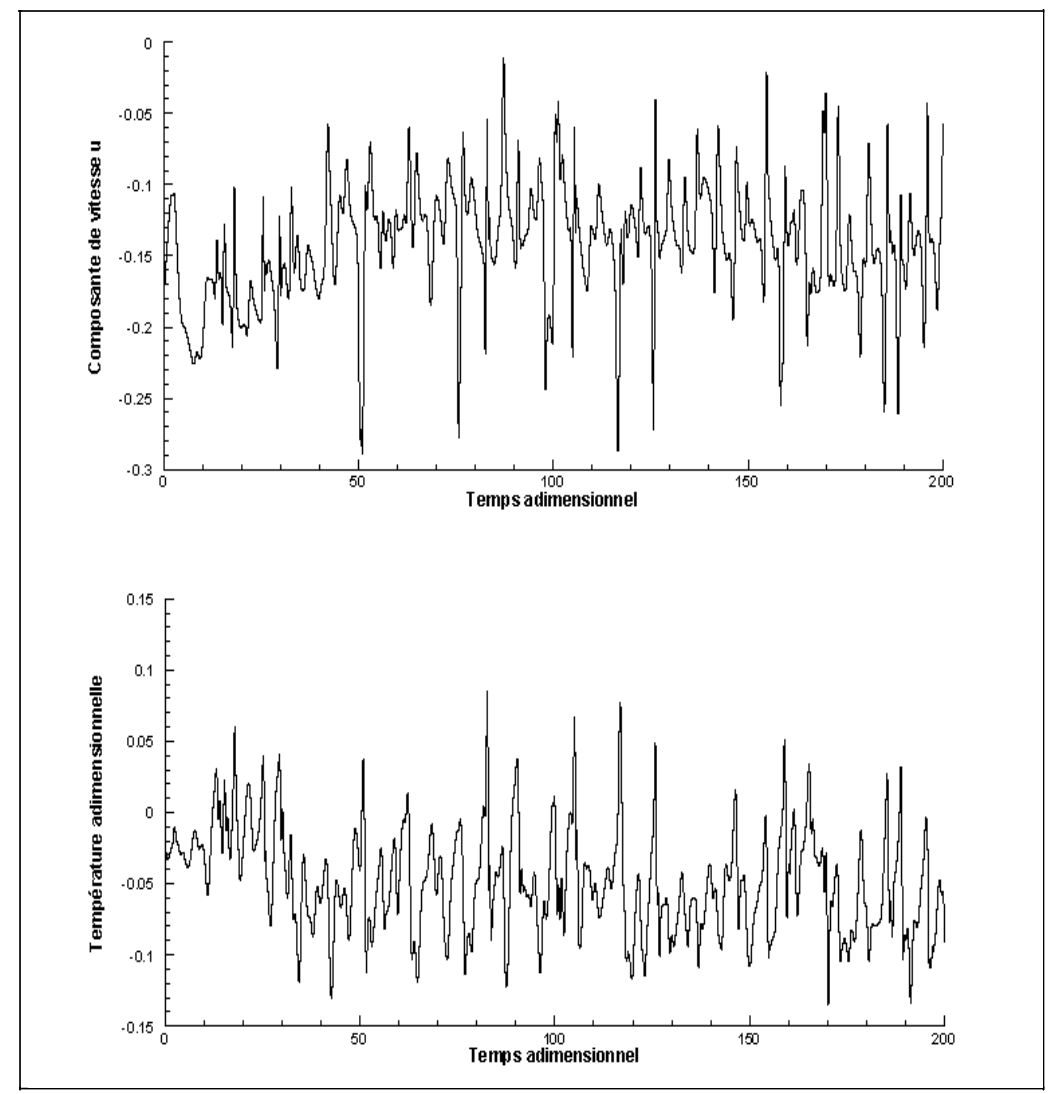

Fig.4. Temporal profiles of both the $\mathrm{u}$-velocity component and the dimensionless temperature for $\mathrm{Ra}=10^{7}$.

As revealed by the Fig4 presented for a temporal interval $(0,50)$, one can see that the profile of both the u-velocity component and the dimensionless temperature versus the dimensionless time are unsteady and exhibit a non-established trend. However, in the time interval between 50 and 200, we may note that the flow seems to become more or less established. 


\section{Effect of Rayleigh number (for $200 \leq \mathrm{t} \leq 600$ )}

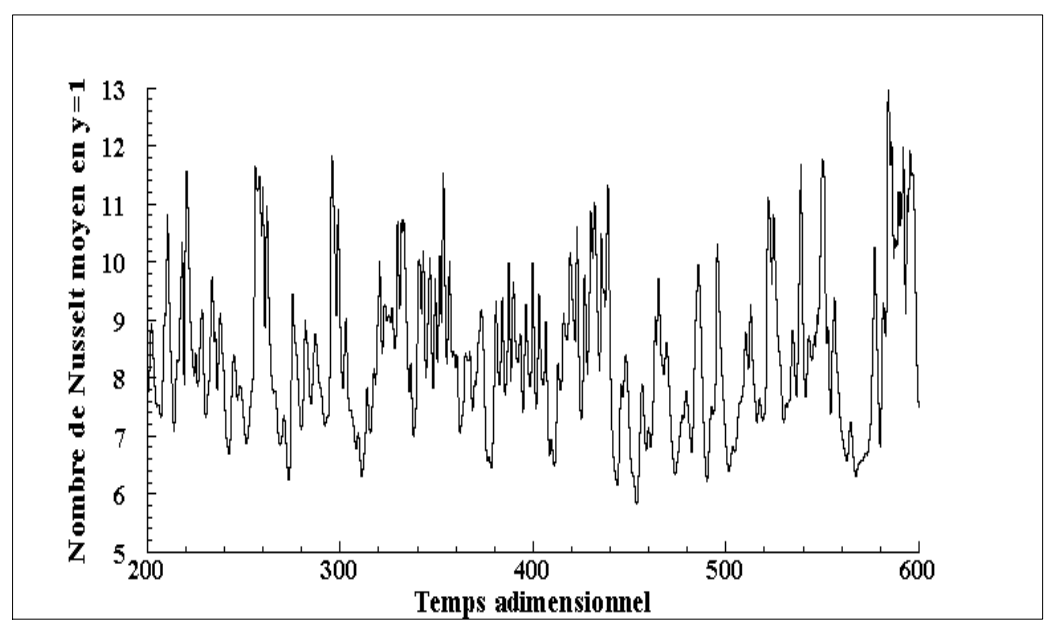

Fig. 5. Temporal evolution of the average Nusselt number at the mid-plane $(\mathrm{y}=1)$ for $\mathrm{Ra}=10^{7}$.

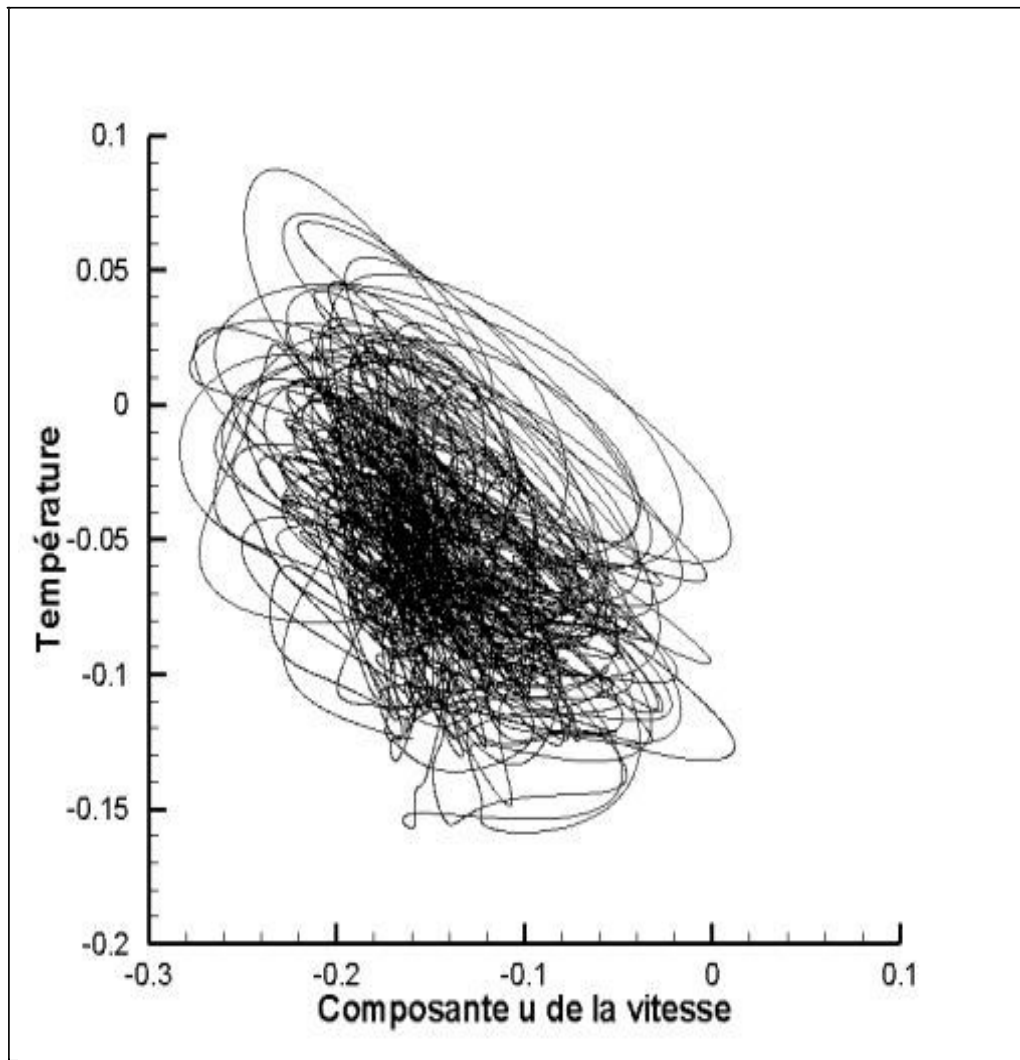

Fig. 6. Phase diagram of the temperature as a function of the $\mathrm{u}$-velocity component for $\mathrm{Ra}=10^{7}$.

The phase diagram describing the evolution of the temperature with respect to the vvelocity component (see fig 6) illustrates multi-closed curves in a very disorderly feature which explains the unsteady disturbed flow. This may explains the existence of multiple frequencies of the velocity component that may induce such a diagram trend. In order to further visualize the flow structure, we illustrate in (Figure 8) the streamlines of the velocity component at 10 instants, $\mathrm{t}_{0}=600, \mathrm{t}_{1}=600.6, \mathrm{t}_{2}=601.2, \mathrm{t}_{3}=601.8, \mathrm{t}_{4}=602.4, \mathrm{t}_{5}=603, \mathrm{t}_{6}=603.6, \mathrm{t}_{7}=$ $604.2, \mathrm{t}_{8}=604.8, \mathrm{t} 9=605.4, \mathrm{t} 10=606$. The results show the appearance of two main vortex 
cells occupying the majority of the cavity and including the obstacle. With time advancing, these vortices seem to be disordered due to the unsteady behavior of the flow structure. Furthermore, $\mathrm{t} 3=601.8$, it is noted the appearance secondary recirculations of localized zones of to the upper horizontal wall of the obstacle. In fact, with further time elapsing, the primary vortices exhibits large deformations and seem to move in a chaotic manner. This is due to the unsteadiness flow pattern that causes intense instability and accordingly affects the structure of the flow within the enclosure.

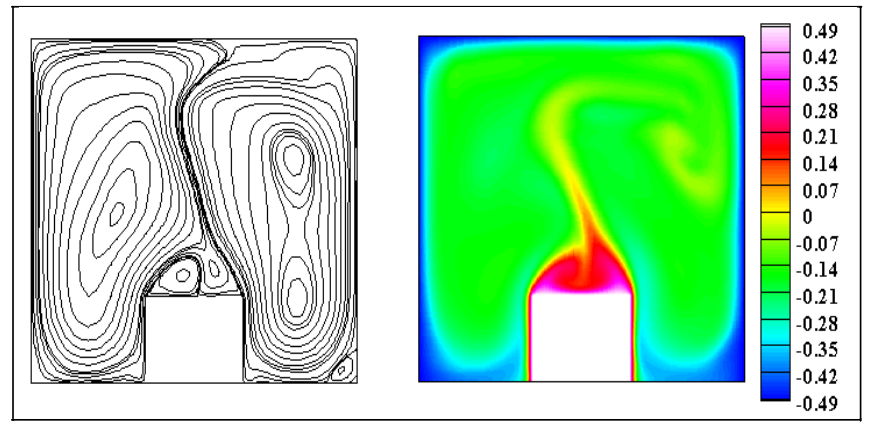

Fig. 7. Structures of isotherms (right) and streamlines (left) for $\mathrm{Ra}=10^{7}$.

Figure 7 illustrates a comparison between the isotherms and streamlines. As illuminated at this figure, the distortion observed at both primary vortices depicted in the streamline plots seem to be in a fair agreement with those described by the isotherms contours.

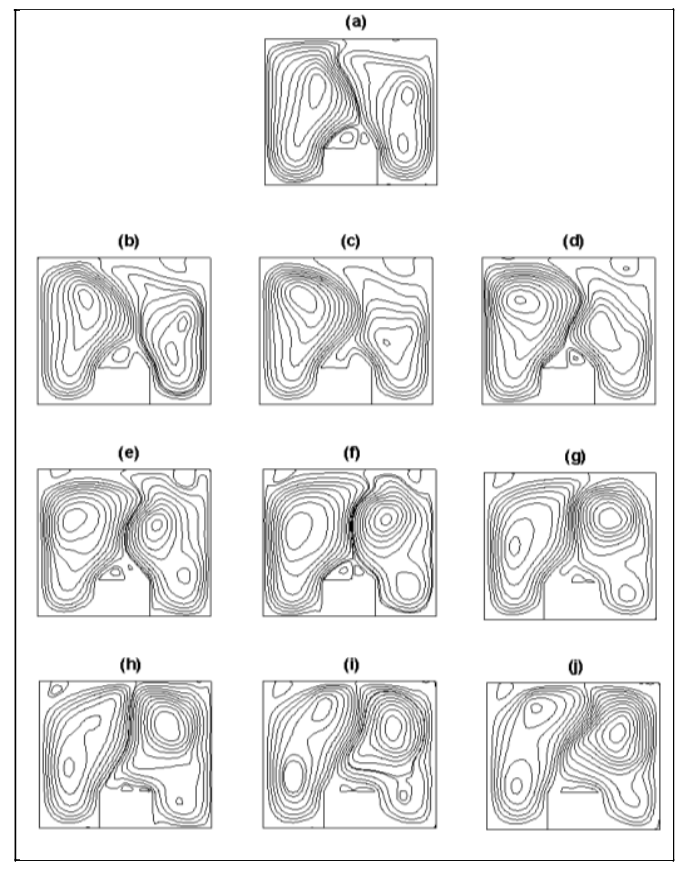

Fig. 8. Streamlines at 10 instants $\mathrm{t} 0=600, \mathrm{t} 1=600.6, \mathrm{t} 2=601.2, \mathrm{t} 3=601.8, \mathrm{t} 4=602.4, \mathrm{t} 5=603, \mathrm{t} 6=603.6$, $\mathrm{t} 7=604.2, \mathrm{t} 8=604.8, \mathrm{t} 9=605.4, \mathrm{t} 10=606 \mathrm{Ra}=10^{7}$

In order to further visualize the flow structure, we illustrate in (Figure 8) the streamlines of the velocity component at 10 instants, $\mathrm{t}_{0}=600, \mathrm{t}_{1}=600.6, \mathrm{t}_{2}=601.2, \mathrm{t}_{3}=601.8, \mathrm{t}_{4}=602.4, \mathrm{t}_{5}$ $=603, \mathrm{t}_{6}=603.6, \mathrm{t} 7=604.2, \mathrm{t} 8=604.8, \mathrm{t} 9=605.4, \mathrm{t} 10=606$. The results show the appearance of two main vortex cells occupying the majority of the cavity and including the obstacle. With time advancing, these vortices seem to be disordered due to the unsteady behavior of the 
flow structure. Furthermore, $\mathrm{t} 3=601.8$, it is noted the appearance secondary recirculations of localized zones of to the upper horizontal wall of the obstacle. In fact, with further time elapsing, the primary vortices exhibits large deformations and seem to move in a chaotic manner. This is due to the unsteadiness flow pattern that causes intense instability and accordingly affects the structure of the flow within the enclosure.

Trend for specific time interval outside of which the flow seems to become more or less stable.

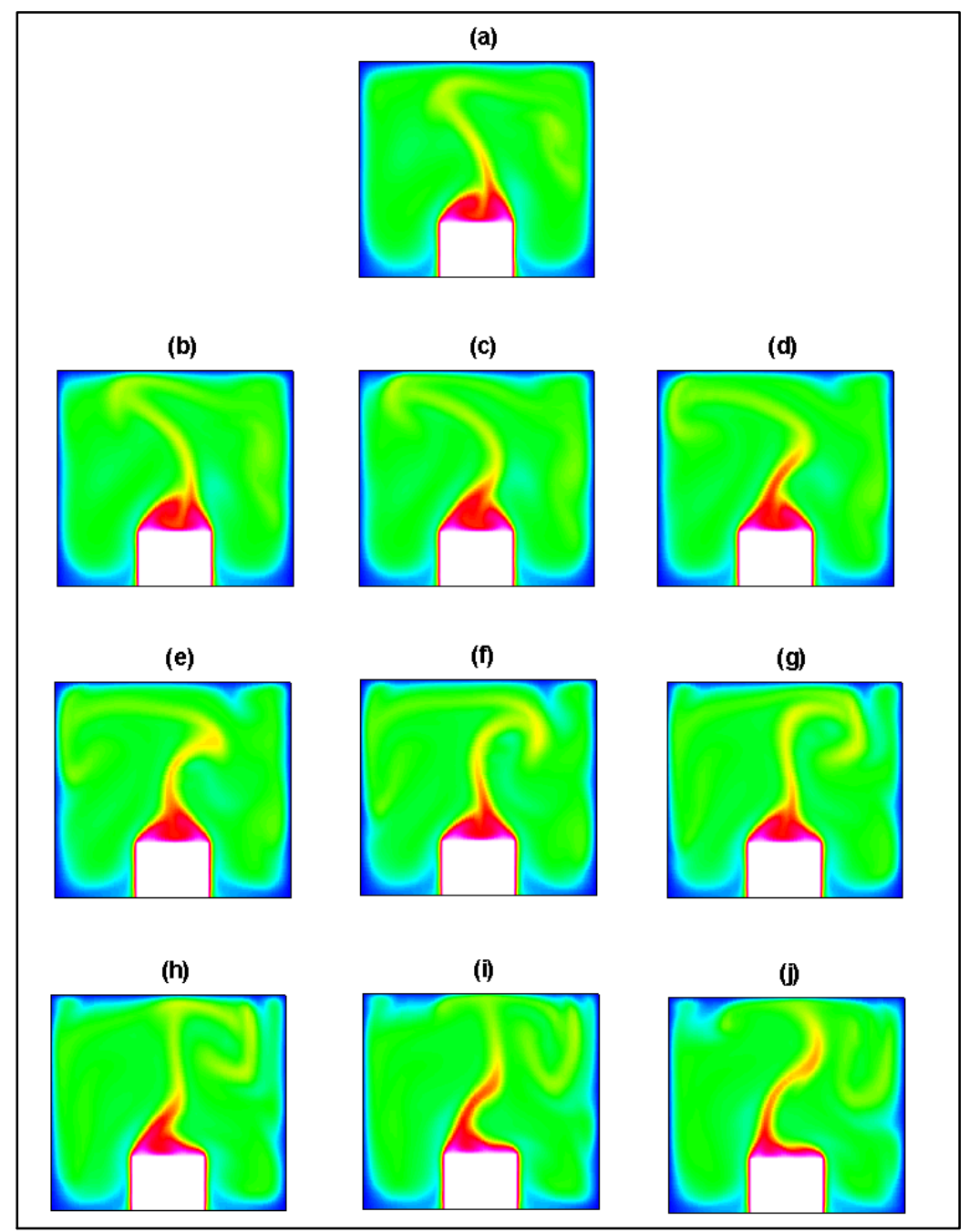

Fig. 9. Isotherms at 10 instants, $\mathrm{t} 0=600, \mathrm{t} 1=600.6, \mathrm{t} 2=601.2, \mathrm{t} 3=601.8, \mathrm{t} 4=602.4, \mathrm{t} 5=603, \mathrm{t} 6=603.6$, $\mathrm{t} 7=604.2, \mathrm{t} 8=604.8, \mathrm{t} 9=605.4, \mathrm{t} 10=606\left(\mathrm{Ra}=10^{7}\right)$.

In Figure 9 are depicted the isotherms at different time instants. As revealed by the figure, these isotherms undergo severe distortion due to the increase of enhancement of the flow rate making the fluid particles accelerated. However, this velocity acceleration is strong enough to trigger the transition to unsteadiness for $\mathrm{Ra}=10^{7}$. It is also noteworthy that this disturbance is manifested in the shape of a cigarette smoke that changes of direction at every time instant.

\section{CONCLUSION}

Natural convection in a cavity with an obstacle placed on its hot wall for Rayleigh numbers ranging from $5 \times 10^{5}$ to $10^{7}$ is investigated in the current study. The effects of Rayleigh number on the unsteady flow pattern have been discussed and presented in terms of the practical 
visualization of streamlines, isotherms and temporal evolutions of Nusselt number. It is noticed that a slight decrease is observed in the extreme values of the stream function due to the presence of a small areas of recirculation occurring at the horizontal wall of the obstacle.

On another hand, it is found that the temporal profiles of both the u-velocity component and the dimensionless temperature remains unsteady and exhibit a non-established trend for specific time interval outside of which the flow seems to become more or less stable.

\section{References}

[1] A. Bazylak, N. Djilali, D. Sinton .Natural convection in an enclosure with distributed heat sources .Numerical Heat Transfer, Part A vol, 49 pp 655-667, (2006).

[2] M. Bakkas, A. Amahmid, M. Hasnaoui, Steady natural convection in a horizontal channel containing heated rectangular blocks periodically mounted on its lower wall, Energy Conversion and Management 47, 509.528, (2006).

[3] H. Bouali, A. Mezrhab, L.Elfarh et CH. Abid, simulation numérique des transferts thermiques dans une serre agricole chauffée par un bloc solide isotherme, 13èmes Journées Internationales de Thermique, France, (2007).

[4] G. Desrayaud, G. Lauriat, Unsteady confined buyoant plumes, J. Fluid Mech. 252, 617-646, (1998).

[5] D. Angeli, A. Pagano, Nonlinear dynamics of a confined buoyant flow, Int. J. Therm. Sci. 2031 (2013).

[6] R. Barrett, etal.Templates for the Solution of Linear Systems: Building Blocks for Iterative Methods, SIAM, (1994).

[7] N. Ben Cheikh, Benchmark solution for time-dependent natural convection flows with an accelerated full-multigrid method, numerical heat transfer 52, 131-151(2007). 